This is the author's copy of the publication as archived with the DLR's electronic library at http://elib.dlr.de. Please consult the original publication for citation.

\title{
The Gnomonic Projection for B-Spline parameterized 4-D Trajectory Optimization Problems
}

\author{
Reiko Müller
}

In aerospace related optimal control problems it is often convenient to parameterize the flight trajectory instead of aircraft control inputs (surface deflections or orientation w.r.t. airflow for instance). This allows to describe the problem with a smaller set of optimizer degrees of freedom, since the kinematical variables representing the trajectory change on a larger timescale and have a smoother shape. One example is the discretization with waypoint positions as parameters, which can then be adjusted by an optimizer to minimize one or multiple criteria. A small parameter set is desirable especially in scenarios with long trajectories (for example intercontinental flights), in order to avoid long computation times and convergence problems. In this work, a new approach for parameterization of such trajectories is presented, which applies the gnomonic projection for generation of shortest-path routes between waypoints. These trajectories feature on the one hand maneouvering phases during departure and approach, and on the other hand long and steady cruise segments, hence necessitating a variable density parameterization approach. Basis splines are first generated in the projected space, subsequently degree-elevated and finally transformed back to ellipsoidal coordinates for computation of derived variables. Ultimately, the method allows to generate realistic 4-D shortest-path trajectories with high continuity, which can be analytically evaluated at every timestep, to serve as reference inputs to a flight control system or an inverse aircraft model.

Keywords: Trajectory Optimization, Optimal control, Parameterization, B-Splines

\section{Copyright Notice}

The author has retained copyright of the publication and releases it to the public according to the terms of the DLR elib archive. Please consult the original publication for citation, see https://arc.aiaa.org/doi/10. $2514 / 6.2020-3205$.

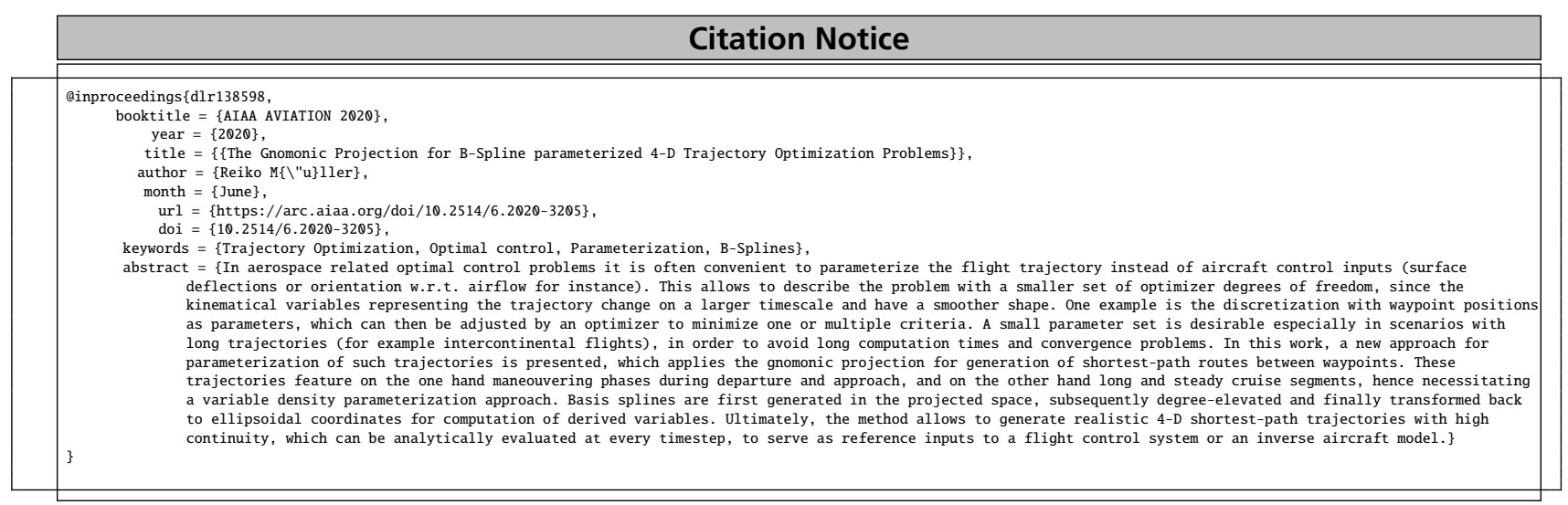

[1] Reiko Müller. The Gnomonic Projection for B-Spline parameterized 4-D Trajectory Optimization Problems. In AIAA AVIATION 2020, June 2020. URL: https://arc. aiaa.org/doi/10.2514/6.2020-3205, doi : 10.2514/6.2020-3205. 


\title{
The Gnomonic Projection for B-Spline parameterized 4-D Trajectory Optimization Problems
}

\author{
Reiko Müller* \\ Institute of System Dynamics and Control, German Aerospace Center (DLR), 82234 Wessling, Germany
}

\begin{abstract}
In aerospace related optimal control problems it is often convenient to parameterize the flight trajectory instead of aircraft control inputs (surface deflections or orientation w.r.t. airflow for instance). This allows to describe the problem with a smaller set of optimizer degrees of freedom, since the kinematical variables representing the trajectory change on a larger timescale and have a smoother shape. One example is the discretization with waypoint positions as parameters, which can then be adjusted by an optimizer to minimize one or multiple criteria. A small parameter set is desirable especially in scenarios with long trajectories (for example intercontinental flights), in order to avoid long computation times and convergence problems. In this work, a new approach for parameterization of such trajectories is presented, which applies the gnomonic projection for generation of shortest-path routes between waypoints. These trajectories feature on the one hand maneouvering phases during departure and approach, and on the other hand long and steady cruise segments, hence necessitating a variable density parameterization approach. Basis splines are first generated in the projected space, subsequently degree-elevated and finally transformed back to ellipsoidal coordinates for computation of derived variables. Ultimately, the method allows to generate realistic 4-D shortest-path trajectories with high continuity, which can be analytically evaluated at every timestep, to serve as reference inputs to a flight control system or an inverse aircraft model.
\end{abstract}

\section{Introduction}

The approach presented in this paper constitutes a new take on the standard way of parameterizing a passenger aircraft trajectory or mission between start and destination airports, when used inside an optimal control problem. These aspects are tackled in the presented approach by a novel B-Spline based, high-continuity parameterization of 4-D trajectories. In addition, the B-Spline are not defined in the obvious ellipsoidal frame of WGS coordinates, but rather in the gnomonic projected space. This allows to easily define distance-minimizing great-circle legs between waypoints which are connected by continuous arc transitions.

In the tradition of Zermelo's method to find wind-optimal routes [1], a lot of research has been performed previously in the context of optimal trajectories for (passenger) aircraft. Approaches which employ projection methods are for example found in [2-4] and [5]. In [2], a planar trajectory under the influence of wind is optimized using the calculus of variations, where the flat-Earth equations are transformed to projected coordinates using the polar stereographic, Lambert and Mercator projections. In the publication by Bijlsma [3], a similar approach is pursued, generating a set of extremal trajectories through the calculus of variations. Girardet et al. [4] consider a wind-optimal planning problem with constant speed that is solved using an ordered-upwind algorithm in the projection space of a Lambert conformal mapping.

Literature concerning 4-D trajectories and their optimization is vast and partly differs in the notion of the 4-D term. An early work of Visser [6] proposes a direct collocation method being employed for optimization of longitudinal mass-point models with constraints on path and along-track distance. Soler et al. [7] optimize a multiphase commercial aircraft trajectory in a stationary wind field by transforming it into a single phase problem with switching times that is solved by collocation. The 4-D aspect is described by combining costs for flight time and fuel consumption into a compound criterion. In [5], a model predictive and an inverse controller are designed for optimal 4-D guidance of atmospheric vehicles. The 4-D reference is computed by previous simulation or interpolation with cubic splines. Another application of the 4-D term arises with the optimization of Continuous Descent Approaches (CDAs), for example by [8], where a 4-D trajectory is calculated between top and bottom of descent with time and groundspeed deviations being eliminated by predominantly elevator and throttle actions to realize a steady CDA. The TEMO (Time

*Research Assistant: Reiko.Mueller@dlr.de 


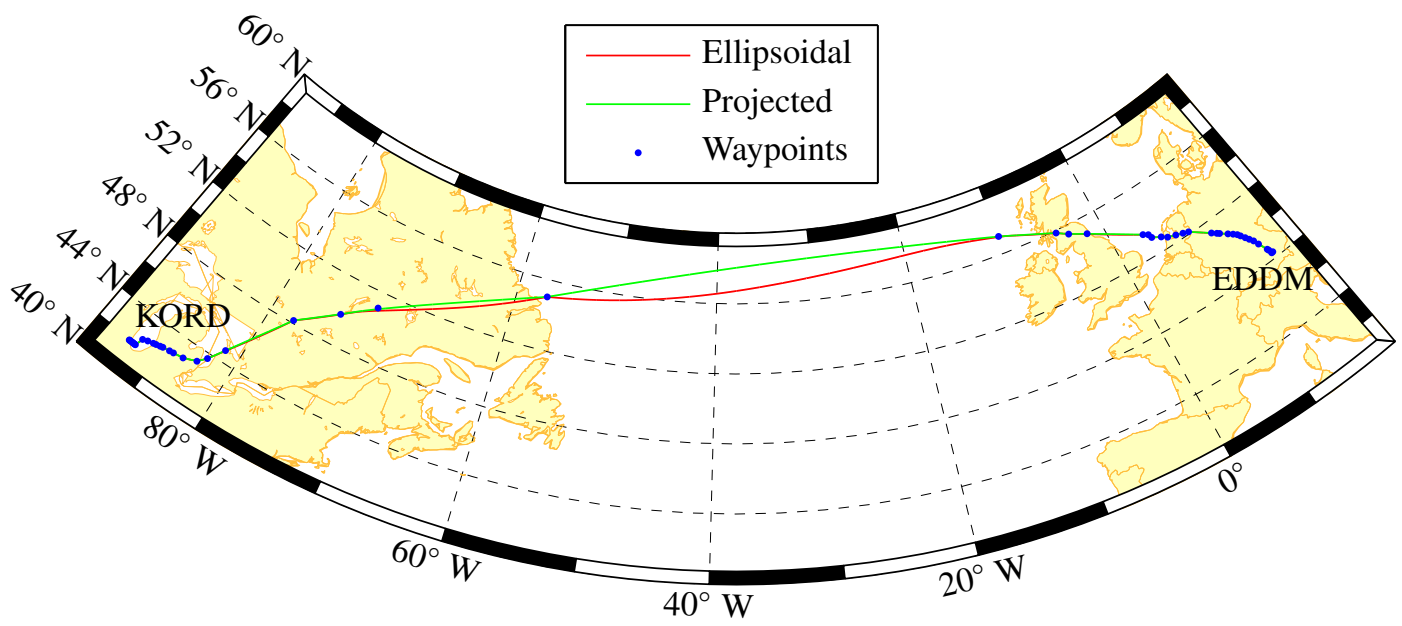

Fig. 1 Comparison of waypoint-based flight trajectories with linearly changing latitude and longitude (ellipsoidal - ) versus the geodesic path gnomonic projection (projected - ), with a shorter overall trajectory length. The gain in distance is higher for longer segments and smaller for shorter legs accordingly.

and Energy Managed Operations) system developed during project CleanSky also allows the 4-D CDA, but with minimized use of speed brakes and throttle during the approach [9]. Foudriat [10] develops an autopilot controller for tracking lateral and longitudinal trajectory based on elliptic integrals and piecewise constant velocity. The velocity is calculated from the remaining time per segment, constituting the 4-D notion.

The parameterization of trajectories for flight guidance and control purposes is frequently performed with Dubins paths [11], polynomials, splines [12] or other curve types like clothoids [13]. Basis-Splines are selected in this context, since they offer several properties that are advantageous for trajectory optimization, for example mathematical robustness, the locality and convex hull properties and easy derivation, and can approximate a standard trajectory consisting of straight legs and arc transitions. By discretizing the trajectory at hand of the waypoints, which are the most common form of defining flight plans, a lower number of optimization tuners is necessary compared to parameterizing the aircraft controls directly. In order to ensure solvability and improve convergence to the optimal solution however, the parameterization function should be of high continuity (for example to allow multiple derivations of the aircraft position to velocities and accelerations). Furthermore, due to the possibly large distances between waypoints, a realistic approximation of actual distance-minimizing trajectories is sought. The actual steps necessary to arrive at such a definition are laid out in Section II.

After the establishment of the 4-D reference trajectory, the multiobjective optimization can be performed, which is described in Section III. The respective aircraft simulation model is outlined in Section III.A, while the actual criterion formulation and optimization setup are given in Section III.B.

Finally, the result of a multiobjective trajectory optimization problem is presented in Section IV. In this example, the new discretization method shows good performance and applicability for increasing the level of detail in optimizing long-range trajectories.

A summary of the presented work and possible future developments are given in the conclusion in Section V.

\section{Trajectory parameterization}

In this section, the trajectory parameterization process is laid out. In the first step described in Section II.A, data needs to be extracted from flight plans (for example provided by Air Navigation Service Providers (ANSPs) or online flight planners) and converted to machine readable format (usually position in ellipsoidal coordinates, velocities, constraints), forming a first set of waypoints. This set is enriched by additional auxiliary waypoints which allow to model different types of leg transitions (for example flyby or flyover). This set of waypoints then is transformed into the gnomonic projection space, described in Section II.B, and quadratic B-Splines are evaluated for position and velocity, yielding linear flight legs and approximate arc transitions. This process is laid out in Section II.C, along with the 
constrained B-Spline approximation routine with integrated degree elevation. As a result, new splines with the same shape, but yielding a higher continuity are obtained. In addition, a spline for the time is determined, which is needed as relation between spline parameter and time vector during the nonlinear simulation. The parameter can then be determined at the current simulation timestep and respective variables are back-projected to the standard ellipsoidal coordinates representing the 4-D trajectory. These variables then form the input to the aircraft or flight controller models described in Section III.

\section{A. Waypoint-based trajectory description}

Aircraft navigation usually relies on constructing a trajectory from navigation fixes which may be installations like Non-Directional Beacons (NDBs) or VHF Omni-Directional Range (VOR) ground stations, but also virtual positions referenced in WGS coordinates. To ensure realism of the optimization setup, the waypoint-based description of flight trajectories is therefore adopted in the proposed setup, more specifically:

- Trajectories consist of several paths that are joined together: Routes like airways and oceanic tracks, user preferred trajectories or airline company routes, Standard Instrument Departure (SID) and Standard Terminal Arrival Route (STAR) procedures, and Instrument Approach Procedures (IAPs).

- Flight plan waypoints serve as point of action for the optimizer, i.e. all degrees of freedom to modify the baseline trajectory are concentrated on these points (see Figure 2, for a graphical depiction of the 4-D Degrees of Freedom (DoFs)).

- Additional or intermediate waypoints may be introduced to further refine the shape of a trajectory, for example adding of turns.

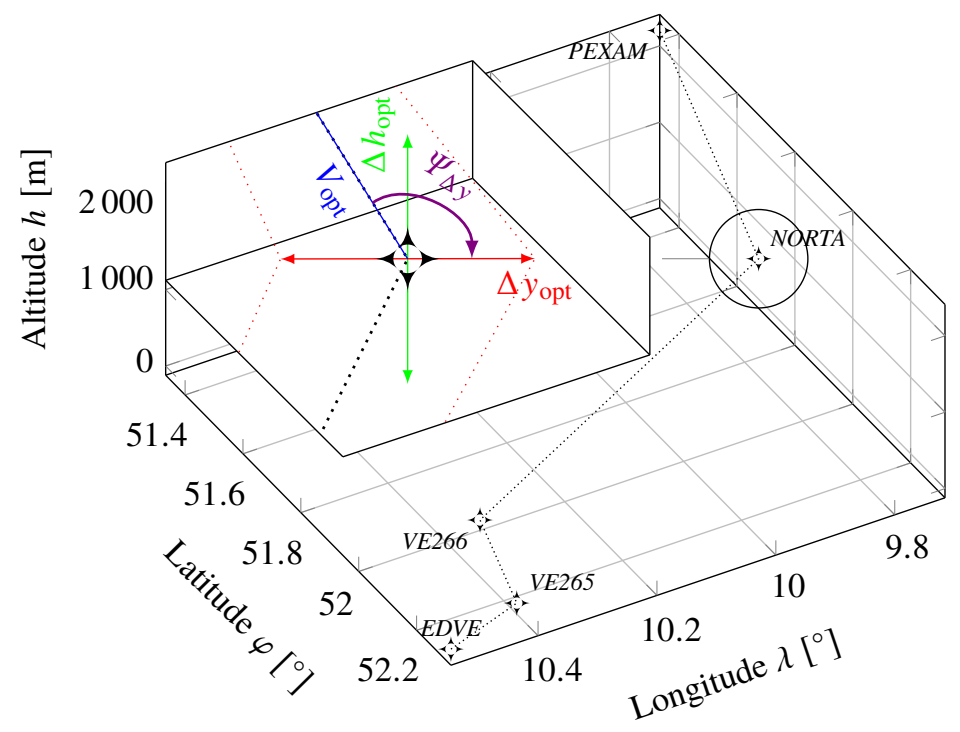

Fig. 2 Optimization Degrees of Freedom (DoFs) / parameters which can be enabled at every waypoint by the user. Altitude and velocity changes are possible inside the bound of $\Delta \boldsymbol{h}_{\mathrm{opt}}$ and $\Delta V_{\mathrm{opt}}$ respectively. Lateral shifting of the waypoint is controlled by $\Delta y_{\text {opt }}$, which points towards the bisecting line between the adjacing flight legs by default. This direction can optionally be modified with the parameter $\Psi_{\Delta y}$.

As is shown in Figure 3, the flight plan waypoints $\mathrm{WP}_{i}$ are treated either as a flyby- (Figure 3a) or flyover- (Figure 3b) waypoint. According to the ARINC 424 [14] specification, all legs are currently modelled as Track to Fix (TF), which is the most important leg type as it defines a geodesic track over ground between two known fixes. During stationary turning with constant roll angle $\Phi$, the lift is equal to $\frac{m g}{\cos (\Phi)}$. Through the centripetal force

$$
m \frac{V_{k}^{2}}{r_{\text {turn }}}=m \dot{\Psi}^{2} r_{\text {turn }},
$$


the relation for determining the turn radius is given:

$$
r_{\text {turn }}=\frac{V_{k}^{2}}{g \cdot \tan (\Phi)}, \quad \text { or } \quad \Phi=\arctan \left(\frac{\dot{\Psi} V_{k}}{g}\right) .
$$

This allows to design trajectories with turn radii adapted to aircraft roll angle constraints (for example $30^{\circ}$ for certain flight states in Airbus aircraft) or defined via standard turn rates $\left(3^{\circ} / \mathrm{s}\right)$. Legacy methods of designing arc transitions by linear and connecting circular segments introduced the so-called turn initiation distance $L_{\text {turn }}$, which is the sum of lengths $l_{1}$ and $l_{2}$ shown in Figure 3:

$$
L_{\text {turn }}=l_{1}+l_{2}=r_{\text {turn }} \cdot \tan \left(\frac{1}{2} \Delta \Psi_{l}\right)+t_{\text {bet }} V_{k}
$$

where $t_{\text {bet }}$ is the bank establishment time (normally set to around five to ten seconds) and accomodates for a smooth transition between zero (linear segment) to nonzero curvature (circular arc). For both flyby and flyover waypoints, the turn initiation distance determines the position of the auxiliary waypoints $\mathrm{WP}_{\mathrm{pre}, i}$ and $\mathrm{WP}_{\mathrm{post}, i}$. In the case of segments with insufficient length, i.e. the remaining distance after subtraction of the two turn lengths $L_{\text {turn }}$ would be smaller than one third of the total segment length, one or both auxiliary waypoint sets are removed. They can also be omitted altogether if there are no geometric constraints on the trajectory at the respective position, and adjacent turn distances can also be set independently (e.g. $L_{\text {turn, inbound }} \neq L_{\text {turn,outbound }}$ ).

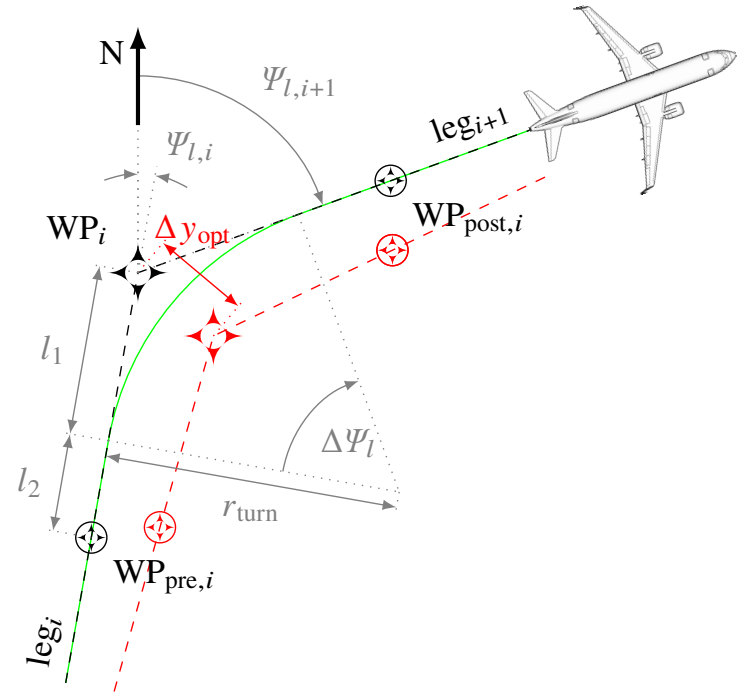

(a) Flyby waypoint geometry, $\mathrm{WP}_{\mathrm{pre}, i}$ and $\mathrm{WP}_{\text {post }, i}$ are located on the two connecting flight legs.

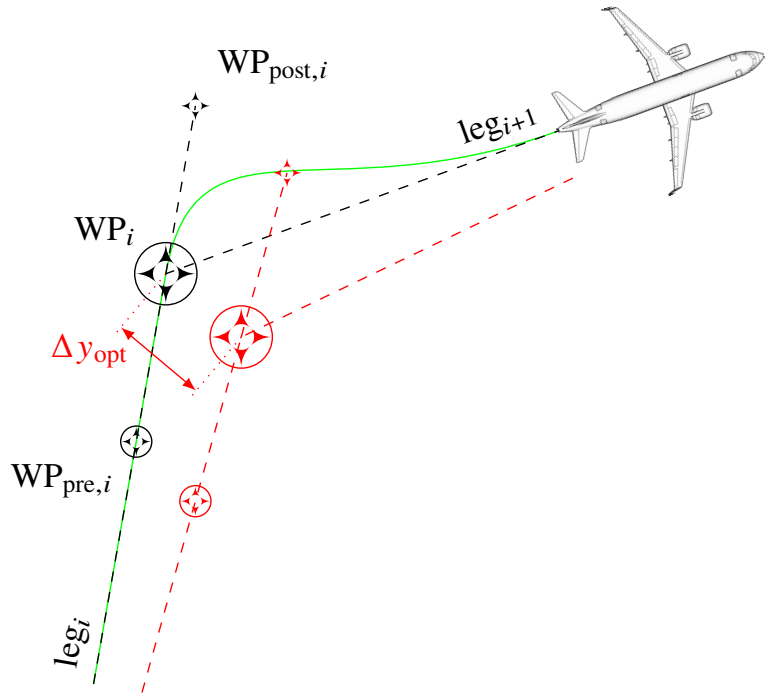

(b) Flyover waypoint geometry, $\mathrm{WP}_{\text {pre, } i}$ and $\mathrm{WP}_{\text {post }, i}$ are located on the colinear extension of the first flight leg.

Fig. 3 Geometry for passing of waypoints, additional waypoints are automatically added to approximate a continuous arc transition. An exemplary shift of the main and auxiliary waypoints (for example due to an optimizer) is shown in red.

When calculating the laterally shifted waypoints for the optimization (shown in red in Figure 3), the direction of the shift is needed. The bisecting angle of the respective adjacent legs is selected as default shift direction as depicted in Figure 3:

$$
\Psi_{\text {bisect }}=\frac{1}{2}\left(\Psi_{l, i}+\Psi_{l, i+1}\right)+\frac{\pi}{2}
$$

Alternatively, the user can also define a radial $\Psi_{\Delta y} \in\left[0^{\circ} \ldots 180^{\circ}\right]$ instead of the bisecting angle, which defines the axis along which the respective waypoint can be moved laterally, with the shift distance $\Delta y_{\text {opt }}$. It is negative for a deviation to the left and positive to the right with respect to the leg heading. For a given radial and its 180 degree complement, it 
has to be determined which one points to the left and right to correctly assign the lateral deviations. For this purpose, the planar vectors $\vec{a}$ and $\vec{b}$

$$
\left.\vec{a}=\left[\cos \left(\Psi_{l, i}\right), \sin \left(\Psi_{l, i}\right)\right)\right]^{\mathrm{T}}, \quad \vec{b}=\left[\cos \left(\Psi_{\Delta y}\right), \sin \left(\Psi_{\Delta y}\right)\right]^{\mathrm{T}},
$$

are stated to form the following product:

$$
q=a_{1} \cdot b_{2}-a_{2} \cdot b_{1} .
$$

$\vec{b}$ is on the left of $\vec{a}$ if $q$ is positive and on the right if negative. For $q=0$, both vectors are parallel. The definition of shifts and their directions allows flexible design of path constraints in the lateral plane.

The 4-D definition also requires that values for inertial velocity $V_{k, i}$ (or according times of arrival) are specified at the waypoint positions $\mathrm{WP}_{i}$ by the user. An alternative is to specify arbitrary velocity or time profiles, which however must lead to trajectories that are flyable by the aircraft. It is assumed in this work, that sets of velocity, altitude and position values originating from standard charts and procedures lead to trajectories which can be flown by the considered passenger aircraft and margins exist which can be used to define reasonable bounds for optimization degrees of freedom. Thus, limits on trajectory / kinematical variables can be modeled via bounds on the control inputs, removing the need for path constraints, that would otherwise have to be satisfied by the optimization algorithm. In the current setup, the velocity values at the $\mathrm{WP}_{i}$ are copied to the auxiliary waypoints $\mathrm{WP}_{\text {pre, } i}$ and $\mathrm{WP}_{\text {post }, i}$

$$
V_{k, i}=V_{k, \text { pre }, i}=V_{k, \text { post }, i}
$$

to ensure that the specified velocity at the main waypoint is attained / that two colinear segments in the velocity frame are obtained (which is necessary for the B-Spline discretization elaborated in the next sections).

\section{B. Shortest-path flight legs with the gnomonic projection}

The emergence of the gnomonic projection is due to the ancient Greeks around the $6^{\text {th }}$ century B.C., who used it for astronomical applications. As an azimuthal projection, lines originating from the projection center always have the same heading. The most significant advantage of the gnomonic projection is that lines defined within the mapping transform back to great-circles in standard spherical coordinates, which allows to find shortest routes between points on a sphere by just linearly connecting them. Besides Mercator projections, it is therefore frequently used for charting and navigation in the maritime and aerospace fields.

Assuming that points with latitude $\varphi$ and longitude $\lambda$ are projected onto a plane, which is tangent to the sphere in a reference point $\left(\varphi_{0}, \lambda_{0}\right)$, through the center of the sphere, the angular displacements in west-east direction $x_{p}$ and south-north direction $y_{p}$ are given by:

$$
\begin{aligned}
x_{p} & =\frac{\cos (\varphi) \sin \left(\lambda-\lambda_{0}\right)}{\sin \left(\varphi_{0}\right) \sin (\varphi)+\cos \left(\varphi_{0}\right) \cos (\varphi) \cos \left(\lambda-\lambda_{0}\right)} \\
y_{p} & =\frac{\cos \left(\varphi_{0}\right) \sin (\varphi)-\sin \left(\varphi_{0}\right) \cos (\varphi) \cos \left(\lambda-\lambda_{0}\right)}{\sin \left(\varphi_{0}\right) \sin (\varphi)+\cos \left(\varphi_{0}\right) \cos (\varphi) \cos \left(\lambda-\lambda_{0}\right)} .
\end{aligned}
$$

For back-conversion to latitude and longitude, the inverse projection equations are used:

$$
\begin{aligned}
& \varphi=\arcsin \left(\frac{\sin \left(\varphi_{0}\right)+y_{p} \cdot \cos \left(\varphi_{0}\right)}{\sqrt{x_{p}^{2}+y_{p}^{2}+1}}\right), \\
& \lambda=\lambda_{0}+\arctan \left(\frac{x_{p}}{\cos \left(\varphi_{0}\right)-y_{p} \cdot \sin \left(\varphi_{0}\right)}\right) .
\end{aligned}
$$

This allows to construct straight line segments on the projected plane that transform back to orthodromes in the spherical Earth modelling. When modeling the Earth as oblate spheroid / ellipsoid, the projection is no longer computable in closed-form as was shown by Karney [15], while only providing a marginal precision gain. To preserve the analytical derivation of Equations (8), (9) and associated ones, the gnomonic projection assuming a spherical Earth is implemented here. In Figure 1, a flight mission or city-pair trajectory from Munich (EDDM) to Chicago (KORD) is depicted, where the red trajectory was parameterized in ellipsoidal and the green one in projected coordinates. The difference is 
especially visible for waypoints which are far apart, for instance between NAT entry and exit points, yielding a $23 \mathrm{~km}$ shorter projected trajectory.

Since derivatives up to second order from latitude and longitude are needed to calculate aircraft model inputs (for example acceleration $\dot{V}_{k}$, course angle rate $\dot{\chi}_{k}$ and pitch angle rate $\dot{\gamma}_{k}$ for a relative degree one inverse model), these must be computed also from the respective derivatives $\frac{\mathrm{d} \varphi}{\mathrm{d} t}, \frac{\mathrm{d} \lambda}{\mathrm{d} t}, \frac{\mathrm{d} h}{\mathrm{~d} t}, \frac{\mathrm{d}^{2} \varphi}{\mathrm{d} t^{2}}, \frac{\mathrm{d}^{2} \lambda}{\mathrm{d} t^{2}}$ and $\frac{\mathrm{d}^{2} h}{\mathrm{~d} t^{2}}$, which are dependent on the projected variables $x_{p}, y_{p}, \varphi_{0}$ and $\lambda_{0}$. For this task, the software MAPLE ${ }^{\circledR}$ or as in this case the MATLAB ${ }^{\circledR}$ Symbolic toolbox can be employed. By according analytical back-projection, the sought variables are computed. For example, assuming an inertial Earth-Centered Earth-Fixed (ECEF) system, the velocity in North East Down (NED) coordinates can then be calculated by:

$$
\vec{V}_{k}=\left[v_{N}, v_{E}, v_{D}\right]^{\mathrm{T}}=\left[\begin{array}{c}
\left(R_{n}+h\right) \cdot \dot{\varphi} \\
\left(R_{e}+h\right) \cdot \dot{\lambda} \cos (\varphi) \\
-\dot{h}
\end{array}\right]=\vec{V}_{\mathrm{tas}}+\vec{V}_{\text {wind }}
$$

with True Air Speed (TAS) $\vec{V}_{\text {tas }}$ and wind speed $\vec{V}_{\text {wind }}$ and the meridional and lateral radii $R_{n}$ and $R_{e}$. The inertial acceleration is found via:

$$
\dot{\vec{V}}_{k}=\left[\begin{array}{c}
\dot{v}_{N} \\
\dot{v}_{E} \\
\dot{v}_{D}
\end{array}\right]=\left[\begin{array}{c}
\left(\dot{R}_{n}+\dot{h}\right) \cdot \dot{\varphi}+\left(R_{n}+h\right) \ddot{\varphi} \\
\left(\left(\dot{R}_{e}+\dot{h}\right) \cos (\varphi)-\left(R_{e}+h\right) \dot{\varphi} \sin (\varphi)\right) \dot{\lambda}+\left(R_{e}+h\right) \ddot{\lambda} \cos (\varphi) \\
-\ddot{h}
\end{array}\right] .
$$

This finally allows to determine the angles $\gamma_{k}, \chi_{k}$ and their derivatives:

$$
\begin{array}{ll}
\gamma_{k}=\arctan \left(\frac{-v_{D}}{\sqrt{v_{N}^{2}+v_{E}^{2}}}\right)=\arcsin \left(\frac{\dot{h}}{\left\|\vec{V}_{k}\right\|}\right), & \dot{\gamma}_{k}=-\left(1-\left(\frac{v_{D}}{\left\|\vec{V}_{k}\right\|}\right)^{2}\right)^{-\frac{1}{2}}\left(\frac{\left\|\vec{V}_{k}\right\| \dot{v}_{D}-v_{D}\left\|\dot{\vec{V}}_{k}\right\|}{\left\|\vec{V}_{k}\right\|^{2}}\right), \\
\chi_{k}=\arctan \left(\frac{v_{E}}{v_{N}}\right), & \dot{\chi}_{k}=\left(1+\left(\frac{v_{E}}{v_{N}}\right)^{2}\right)^{-1}\left(\frac{v_{N} \dot{v}_{E}-v_{E} \dot{v}_{N}}{v_{N}^{2}}\right),
\end{array}
$$

forming the inputs for an inverse aircraft model, or a Flight Control System (FCS) to guide a 3- or 6-DoF aircraft model along the trajectory.

\section{Trajectory discretization using B-Splines}

Parameterization of optimal control problems with piecewise defined polynomials is advantageous, since they can interpolate points without necessarily enlarging the degree of the polynomials. In this work, solely B-Splines are employed for parameterization. They are defined on multiple polynomial segments and either with uniform or non-uniform parameter vector(s). The basic equation for definition of a B-Spline curve is given by:

$$
\boldsymbol{C}(u)=\sum_{i=0}^{n} N_{i, p}(u) \boldsymbol{P}_{i}
$$

with $\boldsymbol{C}$ as the spline value, $p$ being the spline degree, $n$ as number of control points $\boldsymbol{P}_{i}, N_{i, p}$ the $p^{\text {th }}$ degree basis functions and parameter value $u$, which is inside the interval $a \leq u \leq b$ for the knot vector $U$. The knots or vector of breakpoints enable the piecewise definition of the spline with a specified continuity at the segment boundaries via the basis functions:

$$
\begin{aligned}
N_{i, 0} & = \begin{cases}1, & \text { if } u_{i} \leq u \leq u_{i+1}, \\
0, & \text { otherwise. }\end{cases} \\
N_{i, p}(u) & =\frac{u-u_{i}}{u_{i+p}-u_{i}} N_{i, p-1}(u)+\frac{u_{i+p+1}-u}{u_{i+p+1}-u_{i+1}} N_{i+1, p-1}(u) .
\end{aligned}
$$

Their distribution is managed via the knot averaging method, which is described by Piegl and Tiller [16]. The next step for the proposed trajectory description method is the evaluation of the previously established waypoint set (see 


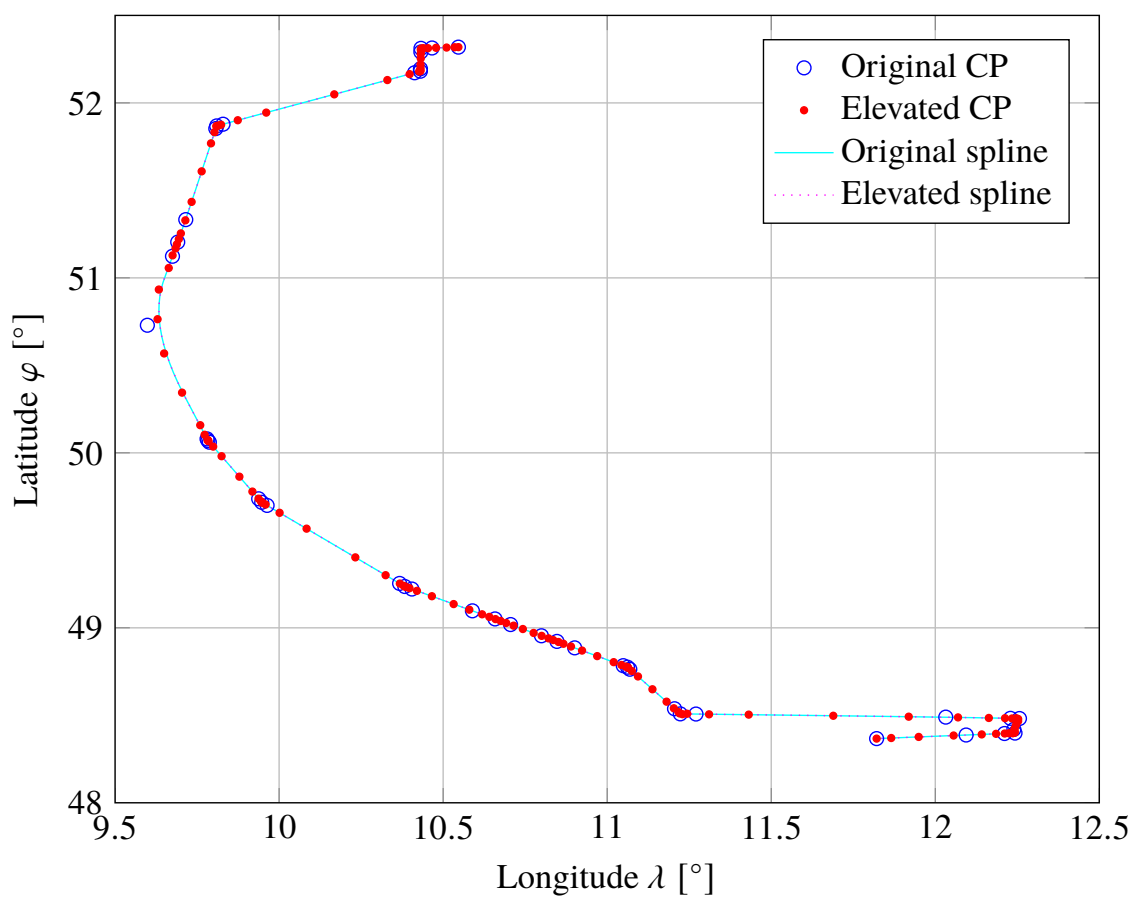

Fig. 4 Comparison of control point distributions for quadratic and quartic B-Splines in the city-pair trajectory between Braunschweig (EDVE) and Munich (EDVE) (also see Figure 2 for the EDVE departure waypoints). More control points are introduced by the degree elevation, along with preservation of shape and higher continuity (no projection was performed in this example for simplification).

Section II.A) in the projected position, altitude and inertial velocity via quadratic spline curves which are all defined over common parameter- and knot vectors (which are in turn distributed according to the control point / waypoint density). These 4-D data points allow to calculate a time-spline (index $u$ refers to the spline associated with the prefix variable):

$$
t_{u}(u)=\int_{u=0}^{u=1} \frac{\mathrm{d} s_{u}(u)}{V_{k, u}(u)} \mathrm{d} u+t_{0},
$$

with $u$ representing the dimensionless spline parameter specified in Equations (12)-(14) and the differential track length:

$$
\mathrm{d} s_{u}=\sqrt{\left(R_{n}+h_{u}\right)^{2} \cdot \dot{\varphi}_{u}^{2}+\left(R_{e}+h_{u}\right)^{2} \cdot \cos ^{2}\left(\varphi_{u}\right) \cdot \dot{\lambda}_{u}^{2}+\dot{h}_{u}^{2}}
$$

The reason for using a quadratic B-Spline is that colinear control polygon segments lead to linear spline segments (see for instance between $\mathrm{WP}_{\text {pre }, i}, \mathrm{WP}_{i}$ and $\mathrm{WP}_{\text {post }, i}$ in Figure $3 \mathrm{~b}$ ), and smooth transitions with a shape similar to a circular arc (see Figure 3a). These then serve as reference for approximating B-Splines with higher degree $p_{a}$ for the same kinematical variables that should ideally have the same shape using a constrained least-squares method described in [16]: With the reference given over the parameter points, and the original control point set $\boldsymbol{P}^{\mathrm{wp}}$, yields the data points $\boldsymbol{Q}$. If derivatives need to be approximated as well, respective test points are calculated additionally, yielding $\boldsymbol{D}$. Unconstrained points in $\boldsymbol{Q}$ and $\boldsymbol{D}$ are combined into $\boldsymbol{S}$, while constrained ones are concatenated into $\boldsymbol{T}$. Accordingly, basis functions belonging to constrained and unconstrained test points or derivatives are combined into arrays $\boldsymbol{N}$ and $\boldsymbol{M}$ respectively. The goal of the constrained least-squares method is to minimize the optionally weighted and squared error between data points and the approximating spline and derivatives (if specified):

$$
f=\sum_{k=i_{u}} w_{k}\left\|\boldsymbol{S}_{k}-\boldsymbol{N}\left(\tilde{u}_{k}\right) \boldsymbol{P}\right\|^{2} \rightarrow \min !
$$

with $i_{u}$ as index for unconstrained values, and while fulfilling the constraints:

$$
\boldsymbol{T}-\boldsymbol{M P}=\mathbf{0},
$$


with the new control points $\boldsymbol{P}^{a}$ as unknowns. In Figure 4, resultant $\boldsymbol{P}^{a}$ of a quartic spline are depicted as red points, while the original control points $\boldsymbol{P}^{\mathrm{wp}}$ are shown in blue. In summary, this B-Spline based description allows to construct straight legs connected with arc segments similar to Dubins paths or other formulations, but with a higher continuity than these. This is a significant advantage for generation of smooth aircraft control inputs, which involve multiple derivations of the trajectory variables latitude $\varphi$, longitude $\lambda$, altitude $h$ and inertial velocity $V_{k}$ (see Section II.B), as those can be better tracked by an FCS and also produce less integrator resets or time events. Tests with B-Spline approximation degrees of quadratic (no approximation) up to quintic splines showed better simulation (less time events) and trajectory tracking performance (less deviation) with higher degrees that is however accompanied by a longer calculation times due to the increase in control points and knots. After several tests, quartic degree splines were found to be the best compromise outperforming the quadratic, cubic and quintic approximations.

\section{Simulation and optimization setup}

This section summarizes the approaches taken for modeling the aircraft in Section III.A and trajectory optimization setup in Section III.B.

\section{A. Aircraft and systems modeling}

For modeling the aircraft, the Modelica language is employed [17], along with the Dymola ${ }^{\circledR}$ IDE. This language offers object-oriented and acausal or equation-based modeling and is widely used in research and industry. The equation-based modeling makes it very easy to derive inverse models of given forward models by just prescribing the outputs and letting the equation reording algorithm automatically transform the system in order to solve for the inputs. Currently, several model fidelities are implemented, ranging from the longitudinal-plane Base of Aircraft Data (BADA) version 3 model [18], to mass-point 3-DoF and rigid-body 6-DoF models, which is depicted in Figure 5. The DLR FlightDynamics library $[19,20]$ written in Modelica allows to follow a flexible object-oriented modeling style, with different implementations of, for example, actuation systems to be interchangeable by standardized interfaces.

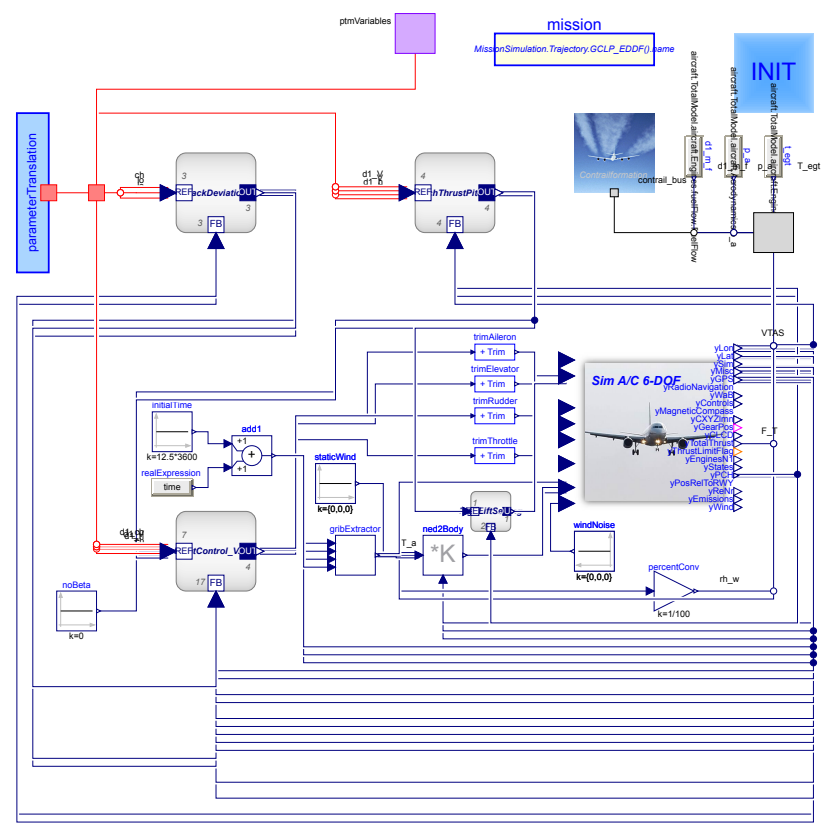

Fig. 5 Modelica implementation of the 6-DoF simulation setup, including weather data and contrail model.

Since the aircraft is required to follow a trajectory reference in position and speed or time, an according 4-D trajectory controller is necessary, which was also used in previous works [21, 22]. It considers aircraft limitations like orientation angles and engine thrust via a Pseudo Control Hedging (PCH) approach and limiters in the flight control system. Along with the DLR Environment library [23], and classes for inclusion of weather data sources (like NOAA GFS and ECMWF ERA data files), comprehensive and realistic simulations can be performed. 


\section{B. Optimization setup}

In modern air traffic, multiple (possibly conflicting criteria) influence the design and execution of a mission. Aside from classical Direct Operating Costs (DOC)-oriented criteria like fuel consumption and operation (flight) time, environmental-related criteria become increasingly important, as the air traffic sector is considered to be a major contributor to global warming. The ACARE FlightPlan2050 agenda for instance lists and recommends future means to protect the environment and energy supply (for example reduction of emissions in $\mathrm{CO}_{2}$ by $75 \%, \mathrm{NO}_{\mathrm{x}}$ by $90 \%$ and perceived noise by $65 \%$ in relation to year 2000 values).

Apart from large scale studies involving long time-frames and aircraft fleets as for example conducted in [24], this trajectory optimization method targets at singular aircraft missions with a higher resolution in the trajectory and aircraft modeling. This approach allows to better judge the gains arising from individual aircraft operation, for example during a city-pair mission, but also for the design of optimized standard routes in airport vicinity (e.g. Standard Terminal Arrival Routes (STARs) and Standard Instrument Departures (SIDs)). According estimates for the production of emissions relevant for air quality and climate effect, namely $\mathrm{CO}_{2}, \mathrm{CO}, \mathrm{NO}_{\mathrm{x}}$ and soot particles (sulfur oxides $\mathrm{SO}_{\mathrm{x}}$ and water $\mathrm{H}_{2} \mathrm{O}$ are no outputs of the model), are obtained from an engine emissions model, that is based on descriptions in [25]. An according simulation model yields emission indices EI for the respective compound $X$ which are then integrated to form total emission masses via:

$$
m_{X}=\int_{t_{0}}^{t_{f}} \mathrm{EI}_{X}(t) \cdot \dot{m}_{\text {fuel }}(t) \mathrm{d} t .
$$

The atmospheric heating and cooling schedule was found to be influenced by cirrus clouds [26], which in turn can form due to persistent aircraft contrails. In recent reviews [27, p. 2, Fig. 1], aircraft induced clouds consisting of contrail-induced cirrus and persistent contrails are accounted for over half of the aviation-related Radiative Forcing (RF) $\left(\mathrm{CO}_{2}\right.$ about $40 \%$ and $\mathrm{NO}_{\mathrm{x}}$ around 5\%). For the presented optimization scenarios, the development of persistent contrails is hence assumed to be negative and their length is to be minimized. A Modelica model describing an approximated physical contrail formation based on the contrail ice criterion process described by Schmidt and Appleman [28, 29] is implemented.

The optimization problem is set up as a direct single shooting problem with multiple objectives, while the right hand side of the problem:

$$
\dot{\boldsymbol{x}}(t)=\boldsymbol{f}(\boldsymbol{x}(t), \boldsymbol{u}(t), \boldsymbol{p}, t)
$$

with states $\boldsymbol{x}$, controls $\boldsymbol{u}$, parameters $\boldsymbol{p}$ combines the submodels (parameterization, FCS, aircraft, criteria models) into a common Modelica wrapper model that is invoked by the optimization algorithm. Within MATLAB ${ }^{\circledR}$, the actual setup is built by means of a specialized software tool called Multi Objective Parameter Synthesis (MOPS), which has been developed by DLR-SR [30] and is in use since several years among DLR and industrial partners. Apart from handling standard parameter optimization problems, it also features modules for Monte-Carlo analysis, model identification, multi-phase optimal control problems and robust systems design. Several optimization algorithms are available to choose from, ranging from gradient-based to evolutionary algorithms and stochastic search methods.

Due to the multiobjective nature of the example scenario, a genetic algorithm was selected as optimizer, since it offers a better solution spread inside the search space and intuitive visualization of dependencies (e.g. Pareto-optimality) between criteria. As stochastic solvers are usually not very well suited to handle (in-) equality constraints, these are indirectly modeled by the parameter bounds (compare list of waypoint tuners, Figure 2) and also limits in the FCS and by the PCH. Due to the high continuity trajectory definition, the path-following parts of the FCS can achieve a high precision, which is usually in the sub-second domain for the time deviation, and within meters for the position deviation. Simulation is terminated for each evaluation if the sign of:

$$
p_{\text {term }}=\vec{d}_{\mathrm{ac} \rightarrow \mathrm{WP}_{\text {end }}} \cdot \vec{d}_{\mathrm{WP}_{\text {end }-1} \rightarrow \mathrm{WP}_{\text {end }}} .
$$

changes, meaning that the aircraft passes the plane located at the last waypoint with normal vector in the direction of the last flight leg. This allows to state the final 4-D time deviation and can be adapted to arbitrary positions along the trajectory to form for example time-of arrival constraints.

\section{Case study}

The cruise phase plays an important role for long haul flights since it represents the longest part of the trajectory. In addition, constraints on lateral and vertical, as well as velocity are less strict (especially over oceans) compared to the departure and approach phases, hence leaving more room for shifting the optimizer degrees of freedom. Apart from 


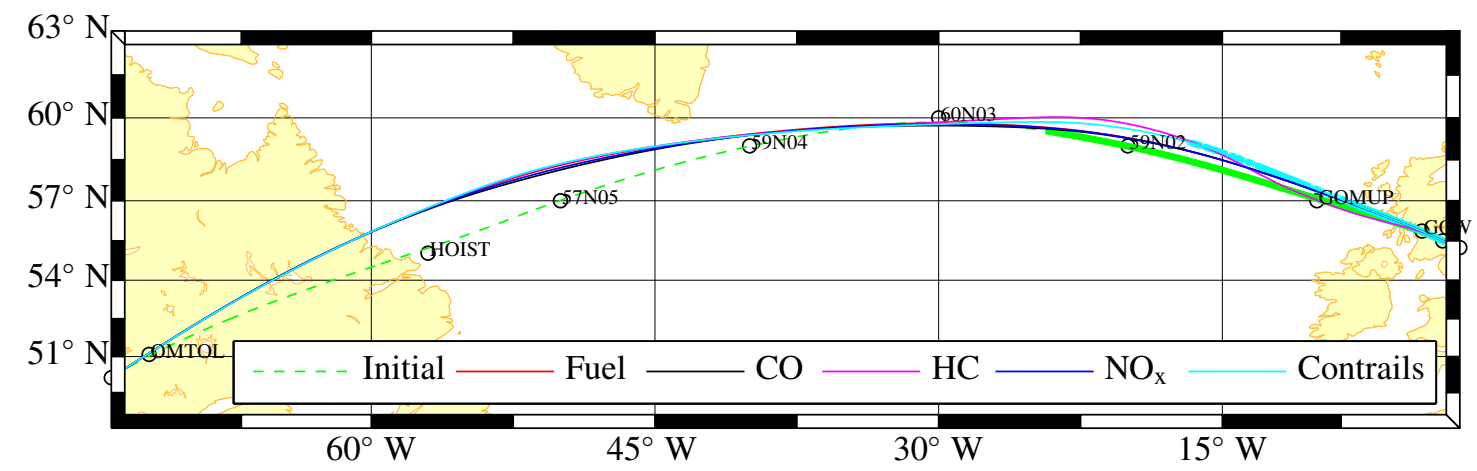

Fig. 6 Cruise phases optimized for various criteria. The thick green and cyan lines show the segments with persistent contrail formation for the initial unoptimized and the minimal contrail solution respectively.

cost-related performance measures like fuel consumption and operating (flight) time, air traffic emissions and their environmental impact are the most important criteria to be influenced by an optimized trajectory. $\mathrm{More}$ specifically, $\mathrm{CO}_{2}$ emissions persist in the upper troposphere, $\mathrm{NO}_{\mathrm{x}}$ emissions lead to heightened formation of ozone and soot particles can promote the formation of contrail-induced cirrus clouds, where all factors contribute to the global greenhouse effect.

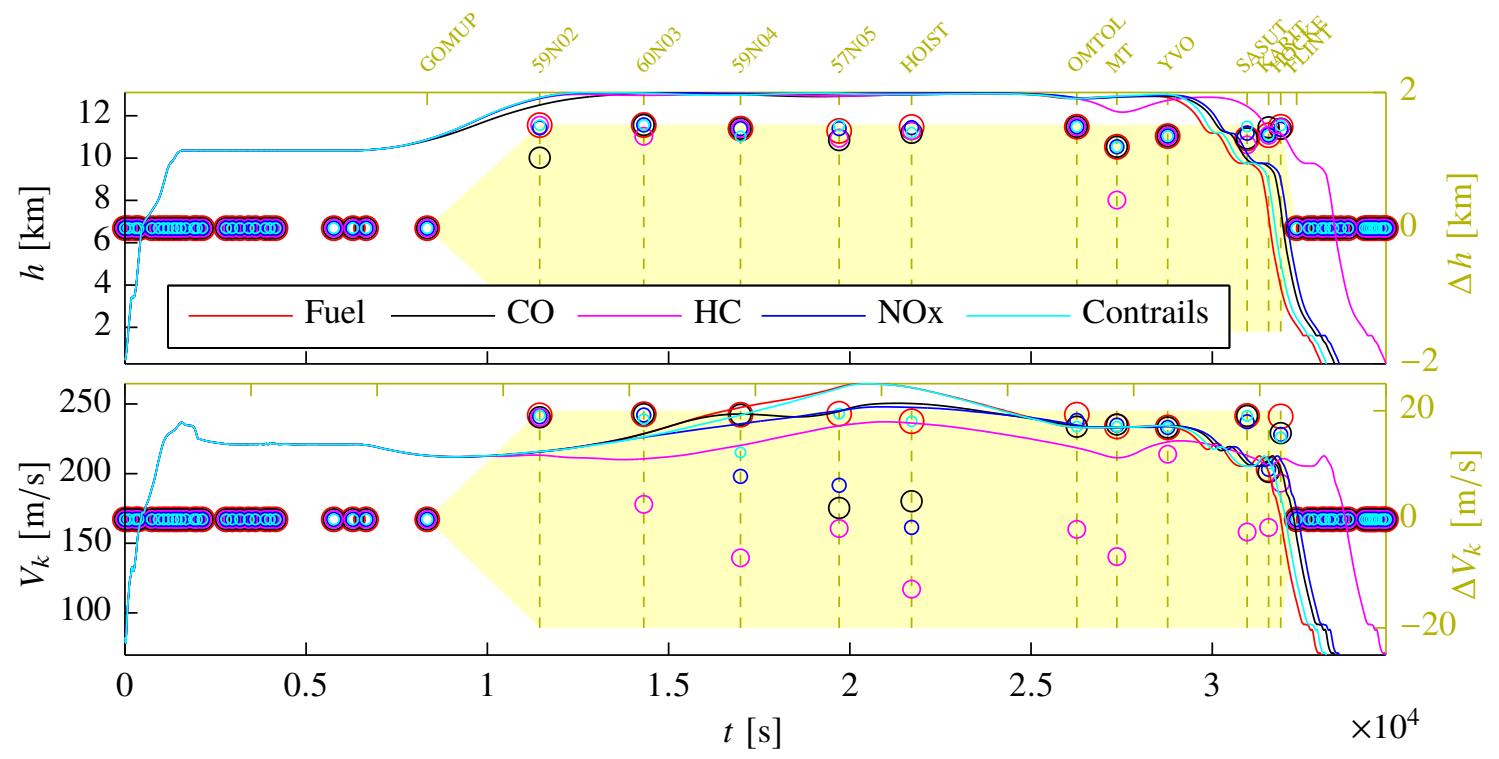

Fig. 7 Longitudinal trajectories and parameter combinations of the extremal criterion solutions. Yellow areas depict the available optimization parameter space for altitude and velocity. Right axes relate to the circled parameter deviations from the baseline trajectory.

As test case for the proposed parameterization algorithm, an optimization of the cruise phase of a transatlantic city pair trajectory between Munich and Chicago airports is considered (see also Figure 1). An aircraft performance model (BADA) of an Airbus A340-600 is implemented in the language Modelica, along with a 4-D capable flight control system and already described trajectory parameterization. Four-dimensional wind is interpolated from an ECMWF ERA Interim [31] dataset in GRIB format at every timestep (as persistent contrail formation is more probable in winter months, an according dataset (3/2/2012) was selected).

Due to the scenario's goal of testing the applicability of the proposed trajectory discretization method and the fact that other traffic was not considered, the usual separation minima in velocity, vertical and lateral plane have been increased: During the North Atlantic Track (NAT), the aircraft is allowed to fly $20 \frac{\mathrm{m}}{\mathrm{s}}$ faster or slower, climb or descend $5000 \mathrm{ft}$ in altitude and deviate $\pm 100 \mathrm{~nm}$ to left or right from the NAT waypoints with respect to the baseline trajectory 

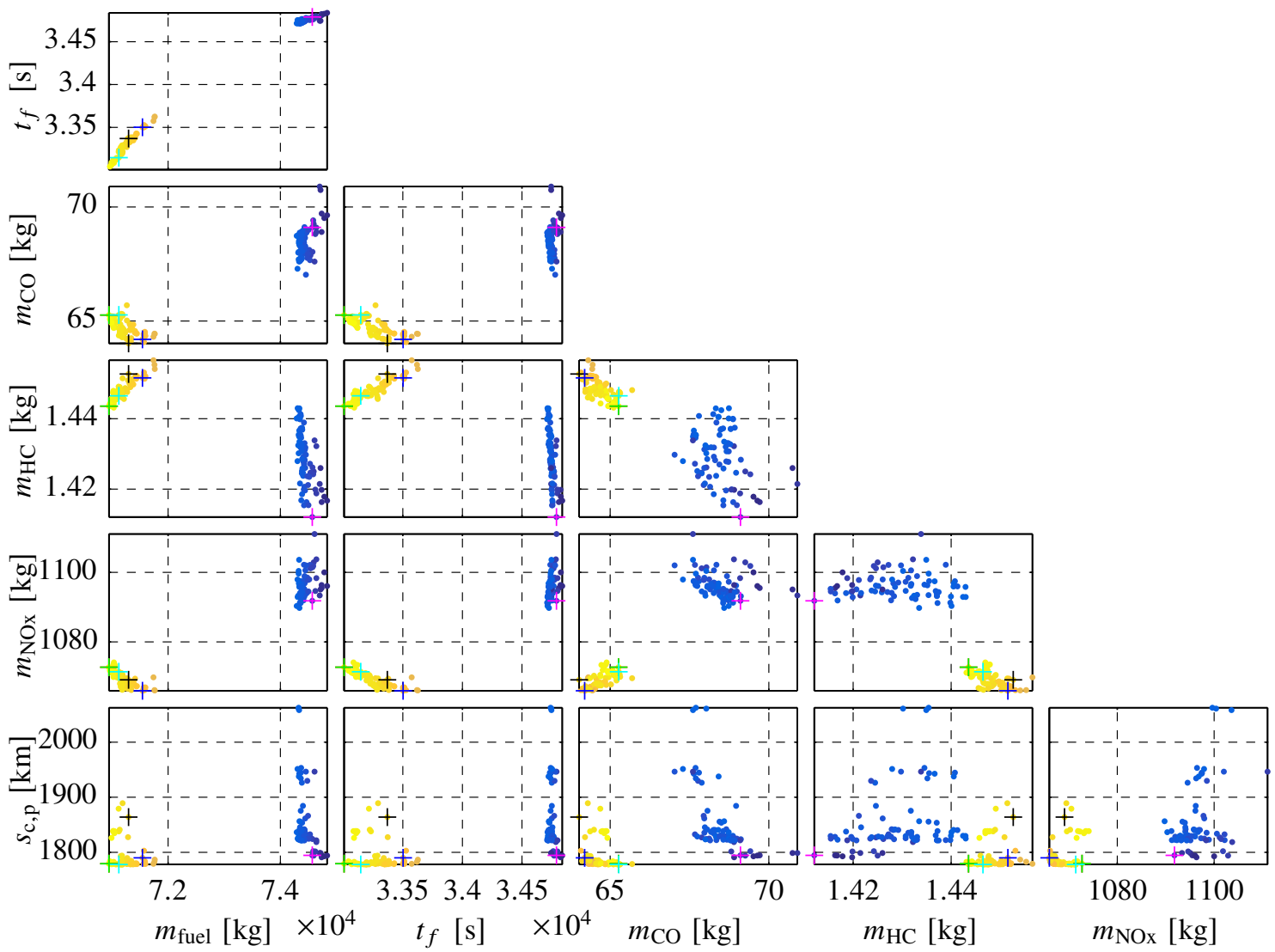

Fig. 8 Candidate solutions for Pareto fronts in the transatlantic city pair trajectory. Extremal solutions are marked for minimum fuel + which is identical to minimum time +, minimum $\mathrm{CO}+, \mathrm{HC}+, \mathrm{NO}_{\mathrm{x}}+$ emissions, and persistent contrail length + .

given in Table 1. In total, 28 optimization parameters were adjusted by a genetic algorithm based on NSGA-2 [32] with a population of 56 individuals for 100 generations. The results of this optimization are shown in Figures 6, 7 and 8 , where the individual extremal solutions lead to improvements of the criteria:

- Fuel consumption $m_{\text {fuel }}: 5.9 \% /$ minus $4510.6 \mathrm{~kg}$

- Flight time $t_{f}: 5.1 \% /$ minus $1758.3 \mathrm{~s} / \approx 29 \mathrm{~min}$

- Carbon monooxide (CO) emissions $m_{\mathrm{CO}}: 8.1 \% /$ minus $5.68 \mathrm{~kg}$

- Hydrocarbons (HC) emissions $m_{\mathrm{HC}}: 4.6 \% /$ minus $68 \mathrm{~g}$

- Nitrogen oxides $\left(\mathrm{NO}_{\mathrm{x}}\right)$ emissions $m_{\mathrm{NO}_{\mathrm{x}}}: 8.3 \% /$ minus $96.5 \mathrm{~kg}$

- Persistent contrail length $s_{\mathrm{c}, \mathrm{p}}: 24.2 \% /$ minus $566.6 \mathrm{~km}$.

In Figure 8, only solutions which are candidates (up to generation 100) for Pareto bounds are depicted, i.e. all other solutions of lower rank are not plotted for better clarity. Fuel and time-optimal trajectories are largely identical (engines run in their most efficient operating point, hence fuel consumption is not so much penalized by the higher speed) and resemble a standard great-circle path. The $\mathrm{NO}_{\mathrm{x}}$ solution is not much different and the contrail-minimal one slightly more northwards in the beginning of the NAT. The HC-optimal trajectory in turn is largely dissimilar from the other extremal solutions, flying relatively slow and therefore increasing overall flight time. For this loosely constrained example, the extremal trajectories show some differences from the intuitive solution of a great circle, mostly due to the atmospheric effects (wind and contrails). When performed with multiple starting conditions (time, date, season, weather), setups like this can help to identify systematic properties of transatlantic flights, such as higher probability of contrail formation or direction of jetstreams in order to improve the routing over these areas. 


\section{Conclusion}

A novel method for parameterization of 4-D high continuity passenger aircraft trajectories is proposed and applied to a testcase. Due to its closed-form B-Spline formulation, derivation and degree elevation of the trajectory is straightforward, and at the same time allows for analytical projection of the trajectory using the gnomonic mapping. The B-Spline approximation with higher order yields smoother trajectory variables and their derivatives, which has benefits on the trajectory tracking precision. The integrated Modelica aircraft model features multiple levels of fidelity to select from and is built up in a modular way to allow for convenient changes in subsystems, for example engine, sensor or actuator models. Detailed environmental models ensure the realistic handling and calculation of optimization objectives. The advantages of the multiobjective formulation are shown in the case study, where different conflicting and concurrent criteria can be weighed a-posteriori by selecting suitable Pareto-front candidate solutions. This parameterization method is not only suited for city - pair trajectories, but can also be applied to analysis and optimization of long- and short term UAV missions (for instance the design of High Altitude Pseudo Satellite (HAPS) climb and descent trajectories) and also shorter segments (for example the shaping of standard approach and departure routes minimizing the effects of air traffic to local airport surroundings), as was shown in previous publications.

\section{Appendix}

Table 1 Baseline trajectory definition for the NAT phase of the city pair trajectory between Munich Franz Joseph Strauss (EDDM) and Chicago O'Hare (KORD) airports.

\begin{tabular}{|c|c|c|c|c|c|}
\hline Waypoint & Latitude & Longitude & Track $\left[{ }^{\circ}\right]$ & Altitude [m] & $V_{k}[\mathrm{~m} / \mathrm{s}]$ \\
\hline GOMUP & N57º0’00.00" & W01000’00.00" & 294.8 & 10668.0 & 210.9 \\
\hline 59N020W & N59 $09^{\circ} 00.00^{\prime \prime}$ & W020 $00^{\circ} 00.00^{\prime \prime}$ & 285.4 & 11582.4 & 195.5 \\
\hline $60 \mathrm{~N} 030 \mathrm{~W}$ & N59 $59^{\prime} 60.00^{\prime \prime}$ & W02959’60.00" & 263.2 & 11582.4 & 205.8 \\
\hline $59 \mathrm{~N} 040 \mathrm{~W}$ & N59 $09^{\circ} 00.00^{\prime \prime}$ & W04000’00.00" & 253.7 & 11582.4 & 226.4 \\
\hline $57 \mathrm{~N} 050 \mathrm{~W}$ & N57º0’00.00" & W05000’00.00" & 246.3 & 11582.4 & 236.6 \\
\hline HOIST & N5501'60.00" & W05700’00.00" & 252.1 & 11582.4 & 252.1 \\
\hline OMTOL & $\mathrm{N} 51^{\circ} 05^{\prime} 00.00^{\prime \prime}$ & W071 ${ }^{\circ} 45^{\prime} 00.00^{\prime \prime}$ & 234.9 & 11582.4 & 221.2 \\
\hline
\end{tabular}

\section{Acknowledgments}

The author would like to thank Andreas Klöckner and Andreas Pfeiffer from DLR as well as Federico Mothes from the University of Applied Sciences Munich for valuable discussions on the presented topics, and Marc May from DLR for providing comments and corrections to the paper.

\section{References}

[1] Zermelo, E., “Über das Navigationsproblem bei ruhender oder veränderlicher Windverteilung," ZAMM - Journal of Applied Mathematics and Mechanics / Zeitschrift für Angewandte Mathematik und Mechanik, Vol. 11, No. 2, 1931 , pp. 114-124. doi:10.1002/zamm.19310110205, URL https://onlinelibrary.wiley.com/doi/abs/10.1002/zamm. 19310110205.

[2] de Jong, H. M., Optimal track selection and 3-dimensional flight planning: theory and practice of the optimization problem in air navigation under space-time varying meteorological conditions, No. 102-93 in Mededelingen en Verhandelingen, Koninklijk Nederlands Meteorologisch Instituut, 1974. URL http://bibliotheek.knmi.nl/knmipubmetnummer/knmipub10293.pdf.

[3] Bijlsma, S. J., "Optimal Aircraft Routing in General Wind Fields," Journal of Guidance, Control, and Dynamics, Vol. 32, No. 3, 2009, pp. 1025-1029. doi:10.2514/1.42425, URL https://doi .org/10.2514/1.42425.

[4] Girardet, B., Lapasset, L., Delahaye, D., and Rabut, C., "Wind-optimal path planning: Application to aircraft trajectories," 2014 13th International Conference on Control Automation Robotics Vision (ICARCV), 2014, pp. 1403-1408. doi:10.1109/ ICARCV.2014.7064521. 
[5] Stiharu-Alexe, I., and O'Shea, J., "Four-dimensional guidance of atmospheric vehicles," Journal of Guidance, Control, and Dynamics, Vol. 19, No. 1, 1996, pp. 113-122. doi:10.2514/3.21587, URL https://doi .org/10.2514/3.21587.

[6] Visser, H., "A 4-D trajectory optimization and guidance technique for terminal area traffic management," Tech. rep., Delft University of Technology, 1994. URL https://repository.tudelft.nl/islandora/object/uuid: 17b2cd01-8c0b4395-aadd-1243f478b427/datastream/OBJ/download.

[7] Soler, M., Olivares, A., and Staffetti, E., "Multiphase Optimal Control Framework for Commercial Aircraft Four-Dimensional Flight-Planning Problems," Journal of Aircraft, Vol. 52, No. 1, 2015, pp. 274-286. doi:10.2514/1.C032697, URL https: //doi.org/10.2514/1.CQ32697.

[8] Garrido-López, D., D’Alto, L., and Ledesma, R. G., "A novel four-dimensional guidance for continuous descent approaches," 2009 IEEE/AIAA 28th Digital Avionics Systems Conference, 2009, pp. 6.E.1-1-6.E.1-11. doi:10.1109/DASC.2009.5347433.

[9] de Jong, P. M. A., de Gelder, N., Verhoeven, R. P. M., Bussink, F. J. L., Kohrs, R., van Paassen, M. M., and Mulder, M., “Time and Energy Management During Descent and Approach: Batch Simulation Study," Journal of Aircraft, Vol. 52, No. 1, 2015 , pp. 190-203. doi:10.2514/1.C032668, URL https://doi .org/10.2514/1. CQ32668.

[10] Foudriat, E. C., “Aircraft 4-D Constant Velocity Control System,” Journal of Aircraft, Vol. 11, No. 6, 1974, pp. $326-333$. doi:10.2514/3.59253, URL https://doi .org/10.2514/3.59253.

[11] Chitsaz, H., and LaValle, S. M., "Time-optimal paths for a Dubins airplane," $200746^{\text {th }}$ IEEE Conference on Decision and Control, 2007, pp. 2379-2384.

[12] Pinchetti, F., Joos, A., and Fichter, W., "Efficient continuous curvature path generation with pseudo-parametrized algebraic splines," CEAS Aeronautical Journal, Vol. 9, No. 4, 2018, pp. 557-570. doi:10.1007/s13272-018-0306-3, URL https: //doi.org/10.1007/s13272-018-0306-3.

[13] Schneider, V., Piprek, P., Schatz, S. P., Baier, T., Dörhöfer, C., Hochstrasser, M., Gabrys, A., Karlsson, E., Krause, C., Lauffs, P. J., Mumm, N. C., Nürnberger, K., Peter, L., Spiegel, P., Steinert, L., Zollitsch, A., and Holzapfel, F., "Online trajectory generation using clothoid segments," 2016 14th International Conference on Control, Automation, Robotics and Vision (ICARCV), 2016, pp. 1-6. doi:10.1109/ICARCV.2016.7838711.

[14] “ARINC Navigation Systems Data Base Specifications 424-18,”, November, 232005.

[15] Karney, C. F. F., "Geodesics on an ellipsoid of revolution,” arXiv preprint arXiv:1102.1215, 2011. URL https://arxiv . org/pdf/1102.1215.

[16] Piegl, L., and Tiller, W., The NURBS book, Springer-Verlag New York, Inc., 1997. doi:10.1007/978-3-642-97385-7, URL https://doi.org/10.1007/978-3-642-97385-7.

[17] Modelica - A Unified Object-Oriented Language for Systems Modeling, Language Specification Version 3.3, Modelica Association, $9^{\text {th }}$ ed., 2014. URL https://www.modelica.org/documents/ModelicaSpec33Revision1.pdf.

[18] EUROCONTROL, "User manual for the base of aircraft data (BADA) revision 3.10," Technical/scientific report no. 12/04/10-45, EEC, 2012

[19] Moormann, D., Mosterman, P. J., and Looye, G., "Object-oriented computational model building of aircraft flight dynamics and systems," Aerospace Science and Technology, Vol. 3, No. 3, 1999, pp. 115-126.

[20] Looye, G., Klöckner, A., Müller, R., Kuchar, R., Re, F., and Leitner, M., “Object-Oriented Aircraft Modelling with the DLR FlightDynamics Library," 63. Deutscher Luft- und Raumfahrtkongress, Deutsche Gesellschaft für Luft- und Raumfahrt, Augsburg, Germany, 2014.

[21] Müller, R., and Looye, G., “A Constrained Inverse Modeling Approach for Trajectory Optimization,” AIAA Guidance Navigation and Control Conference, 2013.

[22] Müller, R., Kiam, J. J., and Mothes, F., "Multiphysical Simulation of a Semi-Autonomous Solar Powered High Altitude Pseudo-Satellite," Aerospace Conference, 2018 IEEE, Big Sky, MT, USA, Institute of Electrical and Electronics Engineers, 2018, pp. 1-16.

[23] Briese, L. E., Klöckner, A., and Reiner, M., "The DLR Environment Library for Multi-Disciplinary Aerospace Applications," Proceedings of the 12th International Modelica Conference, Prague, Czech Republic, May 15-17, 2017, Linköping University Electronic Press, 2017, pp. 929-938. 
[24] Grewe, V., Dahlmann, K., Flink, J., Frömming, C., Ghosh, R., Gierens, K. M., Heller, R., Hendricks, J., Jöckel, P., Kaufmann, S., Kölker, K., Linke, F., Luchkova, T., Lührs, B., van Manen, J., Matthes, S., Minikin, A., Niklaß, M., Plohr, M., Righi, M., Rosanka, S., Schmitt, A. R., Schumann, U., Terekhov, I., Unterstrasser, S., Vazquez-Navarro, M., Voigt, C., Wicke, K., Yamashita, H., Zahn, A., and Ziereis, H., "Mitigating the Climate Impact from Aviation: Achievements and Results of the DLR WeCare Project," Aerospace, Vol. 4, No. 3, 2017, pp. 1-50. URL https://elib.dlr.de/113035/, projekt WeCare.

[25] Deidewig, F., "Ermittlung der Schadstoffemissionen im Unter-und Überschallflug," Scientific report - Deutsches Zentrum für Luft- und Raumfahrt, 1998.

[26] Burkhardt, U., and Kärcher, B., "Global radiative forcing from contrail cirrus," Nature Climate Change, Vol. 1, 2011 , pp. 54-58. URL http://elib.dlr.de/69600/.

[27] Kärcher, B., "Formation and radiative forcing of contrail cirrus," Nature communications, Vol. 9, No. 1, 2018 , p. 1824.

[28] Appleman, H., "The formation of exhaust condensation trails by jet aircraft," Bulletin of the American Meteorological Society, 1953, pp. 14-20.

[29] Schumann, U., “On Conditions for Contrail Formation from Aircraft Exhausts," Meteorologische Zeitschrift, Vol. 5, 1996, pp. 4-23. URL http://elib.dlr.de/32128/.

[30] Joos, H.-D., Bals, J., Looye, G., Schnepper, K., and Varga, A., "A multi-objective optimisation based software environment for control systems design," Proc. of 2002 IEEE International Conference on Control Applications and International Symposium on Computer Aided Control Systems Design, IEEE, Glasgow, 2002, pp. 161-176.

[31] Dee, D. P., Uppala, S. M., Simmons, A. J., Berrisford, P., Poli, P., Kobayashi, S., Andrae, U., Balmaseda, M. A., Balsamo, G., Bauer, P., et al., "The ERA-Interim reanalysis: Configuration and performance of the data assimilation system," Quarterly Journal of the royal meteorological society, Vol. 137, No. 656, 2011, pp. 553-597.

[32] Deb, K., Pratap, A., Agarwal, S., and Meyarivan, T., “A Fast and Elitist Multiobjective Genetic Algorithm: NSGA-II,” IEEE Transactions on Evolutionary Computation, Vol. 6, No. 2, 2002, pp. 182-197. 